\title{
Structural independence of conjugative coupling protein TrwB from its Type IV secretion machinery
}

Delfina Larrea ${ }^{1}$, Héctor D. de Paz ${ }^{1}$, Ignacio Aréchaga ${ }^{1}$, Fernando de la Cruz ${ }^{1}$, and Matxalen Llosa ${ }^{1 *}$

${ }^{1}$ Departamento de Biología Molecular, Universidad de Cantabria (UC), and IBBTEC (UC, CSIC, SODERCAN), Santander, Spain.

*Corresponding author. Departamento de Biología Molecular, Facultad de Medicina, C. Herrera Oria s/n, 39011 Santander, Spain. Phone: +34 942201957. Fax: +34 942201945. e-mail: losam@unican.es

Running title: Structural independence of TrwB from its T4SS

Keywords: coupling protein / Type IV secretion system / plasmid R388 / structural complementation

Abbreviations used: At, Agrogacterium tumefaciens; Bh, Bartonella henseale; Bs, Brucella suis; Bt, Bartonella tribocorum; Dtr, DNA transfer and replication region; T4CP, coupling protein; T4SS, Type IV Secretion System; TRA, transfer region 


\begin{abstract}
The stability of components of multiprotein complexes often relies on the presence of the functional complex. To assess structural dependence among the components of the R388 Type IV secretion system (T4SS), the steady-state level of several Trw proteins was determined in the absence of other Trw components. While several Trw proteins were affected by the lack of others, we found that the coupling protein TrwB is not affected by the absence of other T4SS components, nor did its absence alter significantly the levels of integral components of the complex, underscoring the independent role of the coupling protein on the T4SS architecture. The cytoplasmic ATPases TrwK (VirB4) and TrwD (VirB11) were affected by the absence of several core complex components, while the pilus component TrwJ (VirB5) required the presence of all other Trw proteins (except for TrwB) to be detectable. Overall, the results delineate a possible assembly pathway for the T4SS of R388. We have also tested structural complementation of TrwD (VirB11) and TrwJ (VirB5) by their homologues in the highly related Trw system of Bartonella tribocorum (Bt). The results reveal a correlation with the functional complementation data previously reported.
\end{abstract}




\section{Introduction}

Type IV secretion systems (T4SS) mediate the selective translocation of macromolecules across the cell envelope of Gram negative and positive bacteria. These machines are involved in bacterial functions as different as conjugative DNA transfer and virulence. T4SS can secrete a variety of substrates (protein, DNA, nucleoprotein complexes, or even peptidoglycan) into different target cells (prokaryotic and eukaryotic) or into the milieu (Alvarez-Martínez and Christie, 2009).

T4SS subunits are named after their homologue in the prototypical Agrobacterium tumefaciens (At) T4SS VirB, one of the best studied (Christie and Cascales, 2005); it is composed of 12 proteins named VirB1-VirB11 and VirD4. Although T4SS vary in subunit number and composition, they all seem to have retained a core VirB-VirD-like subcomplex (Christie et al., 2005), and probably assemble and function in a similar manner.

T4SS comprise three types of structural components: i) cytoplasmic ATPases, VirB4, VirB11 and VirD4; the latter is the Type IV coupling protein (T4CP), proposed to link the substrate to be transferred to the T4SS. ii) Integral membrane proteins VirB6, VirB7, VirB8, VirB9 and VirB10, often referred to as core proteins. They span both the inner and outer membranes. iii) The extracellular pilus is composed of a major (VirB2) and a minor (VirB5) component; this structure is believed to mediate contact between cells.

Due to the conservation of T4SS components and structures, it is not surprising that interactions between central components are also conserved. For example the 
interaction between VirB7 and VirB9 has been described for the T4SS in At (Baron et al., 1997; Das et al., 1997), Bordetella pertussis (Farizo et al., 1996), Bartonella henselae (Bh) (Shamaei-Tousi et al., 2004) and Xanthomonas axonopodis (Alegria et al., 2005). Biochemical studies also allowed isolation of VirB7-B9, VirB8-B9-B10, and VirB2B5-B7 subcomplexes; the latter presumably represents pilus preassembly complexes (Krall et al., 2002). Of special relevance was the elucidation of the structures of the VirB7-B9-B10 "core complex" (Chandran et al., 2009; Fronzes et al., 2009) and the recently published image of the complex formed by VirB4 with the core complex (Wallden et al., 2012).

In accordance with the model of conserved structure and function, complementation of heterologous T4SS proteins has been reported in a few instances: At virB1 and virB5 mutations were complemented by their homologues in pKM101 (Schmidt-Eisenlohr et al., 1999b) (Hoppner et al., 2004) and virB1 and virB4, by their counterparts in Brucella suis (Bs) VirB T4SS (Hoppner et al., 2004; Yuan et al., 2005). Several Trw T4SS proteins of plasmid R388 can be substituted in conjugation by their homologues from the related Trw T4SS of Bartonella spp (Bh and Bt); these included TrwD (VirB11) and the core components TrwH (VirB7), TrwG (VirB8), TrwF (VirB9), and TrwE (VirB10) (Seubert et al., 2003) (de Paz et al., 2005).

Based on subunit topologies, 3D structures and biochemical studies, a model for the assembly of a functional VirB-VirD T4SS has been delineated (Waksman and Fronzes, 2010). This model proposes that the core complex forms first; the pilus components VirB2 and VirB5 form a subcomplex, and then pilus biogenesis would require all remaining Vir proteins, except for the T4CP; for substrate secretion, all 
three cytoplasmic ATPases would be required. This model also takes in consideration the results of the DNA translocation pathway delineated by the TrIP assay, which described a temporally and spatially ordered series of substrate-channel subunit interactions (Cascales and Christie, 2004a). The substrate makes sequential contacts with VirD4, VirB11, VirB6/VirB8 and VirB9/VirB2 while it is transferred from the cytoplasm to the outer membrane. The other T4SS components do not interact with the substrate but are essential for substrate transfer at different steps (Cascales and Christie, 2004a).

Coupling proteins (T4CP) are present in all T4SS involved in DNA transfer and also in many T4SS that secrete effector proteins to eukaryotic cells, mediating contact between the T4SS machinery and the transferred substrate. In addition to their coupling role, they may act as DNA pumps during conjugation (Cabezon and de la Cruz, 2006; Llosa et al., 2002). Specific protein-protein interactions with both T4SS and the transferred substrate have been described for the prototype T4CP TrwB of plasmid R388 (Llosa et al., 2003) and VirD4 of At (Atmakuri et al., 2004; Cascales and Christie, 2004a). The interaction of the T4CP with VirB10 has been well established in several DNA-transfer systems [R388, pKM101, R6K (Llosa et al., 2003), R27 (Gilmour et al., 2003), At Ti (Atmakuri et al., 2004)]. T4CP are indispensable for substrate transfer but dispensable for pilus formation (Lai et al., 2000a; Lawley et al., 2002), suggesting that they may be not required for T4SS assembly and structural maintenance. The evolutionary relationship between the T4CP and its T4SS has not been clearly established. Evolutionary studies reported first that conjugative T4CP have co-evolved with their cognate relaxase genes, rather than with their Type IV secretion machineries (Garcillan-Barcia et al., 2009) (de la Cruz et al., 2010). However, a recent report 
compares evolutionary history of T4CP and VirB4 homologues and concludes that T4CP tend to coevolve with both the T4SS and the relaxosome (Guglielmini et al., 2013).

Components of multiprotein complexes often show interdependence for structural stability; in the absence of a functional complex, the observed levels of the unassembled components are lower than in the presence of the complex. In this work we have analyzed a collection of R388 T4SS mutants in order to assess whether the absence of particular proteins alters the steady state level of the other Trw proteins. Our results suggest that the T4CP of R388 does not play a structural role in the T4SS, since TrwB levels were unaffected by any other trw mutation. The effect of Trw mutants in other Trw proteins delineates a possible order of assembly which is similar to the previously described model. We have also tested structural complementation by the Trw proteins of $B t$ and find a correlation with the functional complementation data reported previously in de Paz et al., 2005. 


\section{Materials and Methods}

Bacterial strains and Plasmids. E. coli strain D1210 (Sandler and Clark, 1990) was used as a host for all plasmids. Bacteria were grown in LB (supplemented with agar for solid growth). Antibiotics were added at the following concentrations: ampicillin (Ap), $100 \mu \mathrm{g} / \mathrm{ml}$; chloramphenicol (Cm), $25 \mu \mathrm{g} / \mathrm{ml}$; kanamycin (Km), 25 $\mu \mathrm{g} / \mathrm{ml}$. Plasmids used in this work are described in Table 1. Plasmids were routinely introduced into D1210 by electroporation.

Protein extracts. The amount of Trw proteins was estimated by Western blotting of total protein extracts. D1210 cells harbouring the indicated plasmids were grown in LB broth supplemented with the antibiotic for plasmid selection until $\mathrm{OD}_{600}=$ 0.6; at this point, IPTG was added when indicated. Cells were collected, centrifuged, resuspended in $1 / 10$ volume of $2 X$ SDS-loading buffer, and frozen at -20 으. Samples corresponding to equal amounts of cells (as estimated by optical density) were boiled for 10 minutes and applied to 10\% acrylamide SDS-PAGE gels (Sambrook and Russell, 2001). Protein loading was estimated to be similar in all cases since samples corresponded to a similar number of cells.

Western blots. After the run, gels were transferred to nitrocellulose filters. Gels were stained with $0.1 \%$ Coomassie Brilliant Blue R250 in 50\% methanol to estimate protein transfer and subsequently Trw proteins were detected with specific antisera by Western blot as described in (Towbin et al., 1979). Primary antibodies and dilutions used were: anti-TrwB (de Paz et al., 2010), 1:5,000; anti-TrwC (Grandoso et al., 1994), 1:10,000; anti-TrwD (Rivas et al., 1997), 1:50,000; anti-TrwK (Peña et al., 2011), 1:5,000; anti-TrwJ (Sastre, 1996), 1:10,000. Secondary antibody (peroxidase- 
conjugated anti-rabbit IgG from Sigma-Aldrich) was used at 1:5,000. Detection was performed with the SuperSignal West Pico mol kit (Pierce), using a ChemiDoc ${ }^{\mathrm{TM}}$ System (BioRad); the exposure time was 1,000 seconds. 


\section{Results and Discussion}

\section{R388 T4SS mutants}

The transfer region of plasmid R388 (Figure 1) is divided in two regions named Dtr (DNA transfer and replication) and Mpf (mating pair formation) (Fernandez-Lopez et al., 2006). Dtr includes the oriT, and an operon including the genes that encode the relaxase TrwC, the accessory nicking protein TrwA, and the coupling protein TrwB. Mpf includes all trw genes involved in the formation of the T4SS, plus entry exclusion determinants, and regulator genes. The T4SS genes are proposed to be transcribed in two main operons, trwL-trwl (operon 1) and trwH-trwD (operon 2) (de Paz et al., 2005).

We used a set of previously constructed R388 mutants, each carrying the Tn5tac1 transposon inserted into a different trw gene. For clarity, each gene and protein will be named hereafter using both Trw and Vir nomenclatures, eg. TrwK (VirB4). The non-polar character of Tn5tac1 insertions was previously reported (Llosa et al., 1991), and it was shown that pairs of Tn5tac1 mutants in adjacent genes fully complemented each other (de Paz et al., 2005). Only the trwL (virB2) mutant showed a polar effect on TrwK (VirB4) (S. Bolland, Ph.D. thesis, and data not shown); thus, we have not taken in consideration the results from this mutant. The mutant collection did not include any insertion in trwG (virB8), so we included in our analysis plasmid pSU4035, carrying an insertion of the $\Omega$ interposon in this gene (Bolland et al., 1990). Since this mutant is present in a different replicon (about 5 times the copy number of 
R388), control Western blots were performed to test the levels of several Trw proteins expressed from comparable plasmids based on the two different replicons: pSU4028 (carrying the $\Omega$ interposon in trwA, and thus not affecting the T4SS genes) and pSU2007 (a Km ${ }^{R}$ derivative of R388). The results indicated that the replicon did not affect the observed level of Trw proteins (not shown), allowing us to compare pSU4035 with the rest of R388 trw mutants.

In multiprotein complexes, the absence of one component often affects the stability of the other unassembled proteins. To assess the steady-state levels of Trw proteins in wild-type R388 and trw mutants, we analyzed total protein extracts by western blot with specific antisera. We measured the abundance of proteins from the Dtr region: the T4CP TrwB, and the relaxase TrwC; and proteins from the two operons of the R388 Mpf region (de Paz et al., 2005): TrwJ (VirB5) and TrwK (VirB4) from operon 1, and TrwD (VirB11) from operon 2. The antibodies used allow us to test a representation of the different parts of a T4SS, from cytoplasmic ATPases to pilus components, from the most conserved T4SS element (VirB4) to the most divergent (VirB5).

2. The amount of the T4CP is not affected by the absence of other T4SS components.

TrwC is the R388 relaxase, the protein substrate of the T4SS. It is absolutely required for DNA transfer but dispensable for T4SS assembly and biogenesis. It has no known structural link to the T4SS, so we expected no effect of the T4SS mutants in the cellular amount of TrwC. In fact, we observed that Trw mutants accumulated comparable amounts of TrwC protein (Fig. S1A). Similarly, all trw mutants accumulated 
wild type amounts of the T4CP TrwB (Fig. S1B). This result suggests that the stability of the T4CP does not depend on the existence of a functional T4SS, and argues for a lack of structural dependence between the T4SS and the T4CP.

T4CP are known to interact specifically with their substrates but can interact also with heterologous T4SS with lower affinity (Llosa et al., 2003). To extend the above analysis, we have determined TrwB levels in the presence of different T4SS and relaxosomes. The results are shown in Figure 2 . TrwB was produced in E. coli expressing trwB from the Plac promoter upon IPTG induction; the results shown in the Figure correspond to $3 \mathrm{~h}$ of induction, but identical relative results were obtained after 30 min of addition of IPTG (not shown). Fig. 2 shows that the amount of the T4CP was unaltered by the presence of other plasmid coding for different T4SS (from R388, the related plasmid pKM101, or the Trw T4SS of Bt), or a multicopy plasmid coding for the relaxosomal components of R388 (oriT, TrwA and TrwC) or pKM101 (oriT, TraJ, Tral). Finally, since co-expression of trwA and $\operatorname{trw} B$ gave rise to higher conjugation frequencies (de Paz et al., 2010) and both proteins interact in vitro (Llosa et al., 2003) (Tato et al., 2007), we compared TrwB amounts alone or when coexpressed with trwA; again, no significant differences were observed (Fig. 2).

From these assays we conclude that the presence of a T4SS or a relaxosome of R388 or related systems does not modify TrwB amounts as detected by Western Blot. These results support the proposed role of the T4CP as a third functional module of the conjugative machinery (Llosa and de la Cruz, 2005), which is structurally independent from the substrate and the T4SS. 


\section{Stability of Trw proteins in the absence of other T4SS components}

We have analysed the effect of the absence of each Trw protein on the amounts of other T4SS components: TrwK (VirB4), which is the most conserved element present in all known T4SS, and is known to interact with the core complex (Wallden et al., 2012); TrwD (VirB11), a cytoplasmic ATPase associated with the membrane, involved in both substrate transfer and pilus biogenesis (Sagulenko et al., 2001a); (Cascales and Christie, 2004a); and TrwJ (VirB5), a minor component of the pilus, which is believed to be the last part of the T4SS to be assembled (Waksman and Fronzes, 2010). The results of the Western blots using antisera for these R388 Trw proteins are shown in Figure $\mathbf{3}$ and are compiled in Table $\mathbf{2}$ to facilitate their discussion.

TrwK (VirB4) was detectable in all trw mutants (except for trwL which, as explained above, shows a polar effect on trwK). The intensity of the signal varied: the trwE (virB10) mutant produced an increased amount of TrwK (VirB4), whereas for trwl (virB6), trwF (virB9) and trwG (virB8) mutants, reduced quantities were observed compared to wild-type R388. Mutations in trw genes had a severe effect on TrwD (VirB11) levels; the protein was undetectable in trwF (virB9), trwG (virB8), and trwK (virB4) mutants, while reduced amounts were detected in trwl (virB6) mutant. Mutation in $\operatorname{trwB}$ (the T4CP) caused a modest reduction on TrwD (VirB11) amounts. In contrast, higher amounts were observed for trwH (virB7) and trwJ (virB5) mutants. In the case of TrwJ (VirB5), its levels were completely abolished in all trw mutants encoded in the Mpf region, suggesting that TrwJ (VirB5) needs an assembled T4SS for its own integrity. A mutation in trwB did not change the levels of TrwJ (VirB5). This 
result supports previous works showing that TrwB is not required for pilus production, as inferred by the sensitivity of $\operatorname{trw} B$ mutants to the pilus-specific phage PRD1) (Bolland et al., 1990). Similarly, the T4CP VirD4 of At and TraD of F are not required for pilus biogenesis (Lai et al., 2000a) (Ghosh et al., 2008).

Similar studies have been carried out in the VirB T4SS of $A t$, to test the influence of T4SS mutants in the stability of other T4SS components. Although overall the results reported correlate with ours, there are some significant differences. In At, the absence of VirB4 did not affect steady-state levels of VirB5 and VirB11 (Yuan et al., 2005), while R388 TrwK (VirB4) mutant produced undetectable levels of TrwD (VirB11) and TrwJ (VirB5). It was also reported that the absence of VirB11 did not influence the amount of pilus subunits VirB2 and VirB5 (Sagulenko et al., 2001a). In our case, no TrwJ (VirB5) was detected in the absence of TrwD (VirB11) (Fig. 3D). Other study reported that virB6 mutant did not change levels of Vir proteins except for VirB5 (Hapfelmeier et al., 2000) while we found low TrwK (VirB4), very low TrwD (VirD11), or undetectable TrwJ (VirB5) protein levels caused by the trwl (virB6) mutation. Finally, an At virB7 mutant produces decreased amounts of several VirB proteins including VirB11 and VirB4 (Fernandez et al., 1996b); in the R388 system, the trwH (virB7) mutant showed an increased amount of TrwD (VirB11) and wild-type levels of TrwK (VirB4). All these differences may reflect a different pattern of protein-protein interactions in different T4SS, or more likely, a different effect on T4SS protein stability in the absence of an assembled T4SS. 


\section{Structural complementation of R388 trw mutants by the Bt Trw T4SS}

In previous reports, we demonstrated the existence of functional interactions among R388 Trw proteins and their homologues in the Trw T4SS of Bt (de Paz et al., 2005). The R388 mutants trwH (virB7), trwG (virB8), trwE (virB10) and trwD (virB11) were functionally exchangeable to different extents (Table 2 col. 3) (de Paz et al., 2005). Even in the absence of functional complementation, when a protein from one T4SS is able to substitute structurally for its homologue in another T4SS, this may allow system assembly and therefore revert the destabilizing effect of the absence of the cognate protein. As shown in the above section, TrwD (VirB11) and TrwJ (VirB5) were severely affected in the absence of other Trw proteins, allowing to assay structural complementation by their homologue Trw proteins of the Bt T4SS and to compare with previous functional complementation data. Plasmid $\mathrm{pAB} 2$, coding for the $B t$ Trw T4SS, was introduced in cells carrying the R388 Trw mutants, and Western blots were performed in parallel with cells not carrying pAB2.

As shown in Fig. $3(B, C)$, endogenous TrwD (VirB11) levels were not reconstituted in most R388 trw mutants in the presence of $B t$ T4SS except for a weak signal detected for trwK (virB4) mutant, indicating structural complementation by $B t$ VirB4. This protein shares around $80 \%$ amino acid identity with R388 TrwK, however it cannot substitute it in conjugation (de Paz et al., 2005), suggesting that the $B t$ protein can substitute that of R388 into the T4SS scaffold, but lacks specific interactions needed for substrate translocation. In the case of $A t$ and Bs VirB T4SS, both structural and functional complementation was observed between their VirB4 components (Yuan et al., 2005). We also found that Bt TrwH (VirB7) reverts the effect of the R388 trwH mutation, causing a decrease to wild-type levels of TrwD (VirB11) (Fig. 3 B, C, lane 5), 
in accordance with the previously observed functional complementation (Table 2). In the presence of the Bt T4SS, TrwJ (VirB5) levels were recovered in R388 trwH (virB7), trwG (virB8), trwF (virB9), trwE (virB10) and trwD (virB11) mutants (Fig. $3 \mathrm{D}, \mathrm{E})$, correlating perfectly with the functional complementation data described previously [Table 2 col.3; (de Paz et al., 2005)].

\section{Assembly model for the T4SS of plasmid R388.}

The assembly model for At and pKM101 VirB T4SS proposes that the core complex made of VirB7 -VirB9 -VirB10 forms in the inner membrane and inserts into the outer membrane. That structure would then nucleate the formation of the translocation apparatus by the addition of VirB6 and VirB8 at the inner membrane, and VirB4 at the cytoplasm; then pilus subunits VirB2 and VirB5 would join to form a minimal T4SS functional unit. Finally, the ATPases VirB11 and VirD4 would add in the cytoplasm (Waksman and Fronzes, 2010). In accordance with this model, we observed that the absence of Trwl (VirB6), TrwG (VirB8) or TrwF (VirB9) affect the steady-state levels of TrwK (VirB4) and TrwD (VirB11). As already discussed, in our case the absence of TrwH (VirB7) does not alter the stability of other T4SS components.

We propose a tentative assembly pathway for the R388 T4SS based on the previously proposed model (Waksman and Fronzes, 2010), with modifications suggested by our results:

- In a first step, the cytoplasmic ATPase TrwK (VirB4) would be anchored to the inner membrane establishing interactions with components of the core complex, as shown by (Wallden et al., 2012). We observed that the absence of TrwE (VirB10) 
does not affect stability of other T4SS components, suggesting it is assembled after the rest of the core components.

- Our results suggest that the pilus is assembled in the last place, as reported by others, since the integrity of the minor pilus component TrwJ (VirB5) is dependent on all T4SS components except for the T4CP TrwB (VirD4). Interestingly we found that trwH (virB7) and trwJ (virB5) mutants caused TrwD (VirB11) accumulation. VirB7 forms part of pilus preassembly complexes (Krall et al., 2002). It is possible that a pool of TrwD (VirB11) exists free in the cytoplasm or associated to the inner membrane depending on the stage of the T4SS assembly.

- The T4CP is structurally independent from other T4SS proteins. We suggest that TrwB is anchored to the inner membrane as a monomer, and when a mating signal is detected, it would locate at the T4SS to recruit the substrate. After protein translocation, the T4CP would oligomerize around the single stranded DNA and pump it out of the cell using the energy obtained from ATP hydrolysis, as previously proposed (Cabezon and de la Cruz, 2006; Llosa et al., 2002).

\section{Acknowledgments}

This work was supported by grant BIO2010-11623-E to ML. Work in FC lab was supported by Spanish Ministry of Education (BFU2011-26608), and European VII Framework Program grants no 248919/FP7-ICT-2009-4 and 282004/FP7HEALTH.2011.2.3.1-2. DL was a recipient of a JAE-PRE predoctoral fellowship from the CSIC (Spain). 


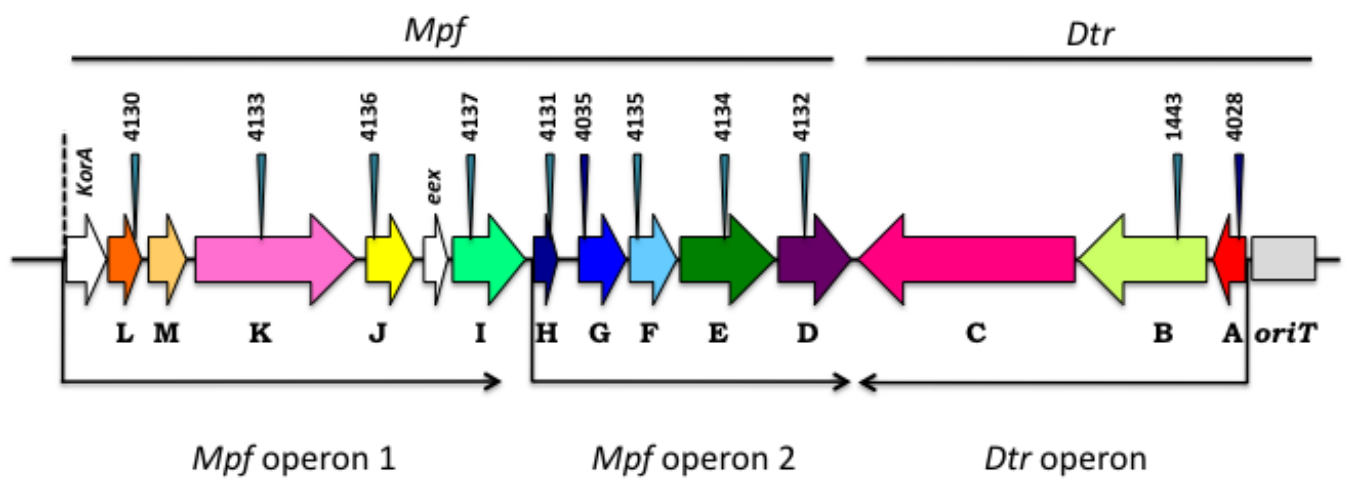

Figure 1. The R388 transfer region. The extent of the Mpf and Dtr regions is indicated with thick black horizontal lines. The Trw ORFs are indicated with coloured arrows and named by their last letter (Trw prefix omitted for clarity). Proposed operons are indicated with black arrows. The vertical arrowheads indicate the site of insertion of Tn5tac1 (light blue) or $\Omega$ interposon (dark blue) in the indicated R388 mutants (pSU prefix omitted). 


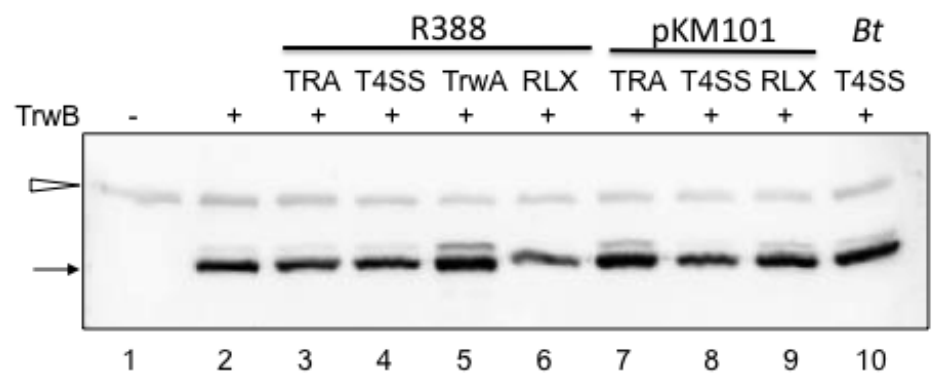

Figure 2. Western blots with anti-TrwB. Samples are total extracts from D1210 cells containing the indicated plasmids, which code for $\operatorname{trw} B$ under the Plac promoter, and different components of the R388 or pKM101 transfer machineries, or Bt T4SS. TRA: complete transfer region; in the case of R388, this was provided from an R388 trwB mutant (pSU1443), so the only source of TrwB was the expression vector. RLX= relaxosomal components (oriT + relaxase + accessory protein). Description of the plasmids used and their replicons can be found in Table 1. Line 1, no plasmid; line 2, pHP139; line 3, pSU1443+ pHP139; line 4, pSU4058 + pHP139; line 5, pDEL003; line 6, pHP139 + pHP138; line 7, pKM101 + pHP139; line 8, pKM101 2 mob + pHP139; line 9, pMTX539 + pHP139; line 10, pAB2 + pHP139. The black arrow points to TrwB. The white arrowhead points to a nonspecific band. 




Figure 3. Western blots to detect TrwK, TrwD and TrwJ. Total extracts of $E$. coli cells containing R388 derivatives were processed as described in Materials and Methods. Samples were run on SDS-PAGE, and proteins were detected with A) antiTrwK $(\alpha K), B, C)$ anti-TrwD $(\alpha D), D, E)$ anti-TrwJ $(\alpha J)$. In C) and E) cells were cotransformed with pAB2 cosmid expressing the Bt Trw T4SS. At the top of the gel, the Trw mutant is indicated. The black arrows indicate the protein detected specifically. The white arrowhead points to nonspecific bands visible in the strain with no plasmids. 


\section{Supplementary material}

A

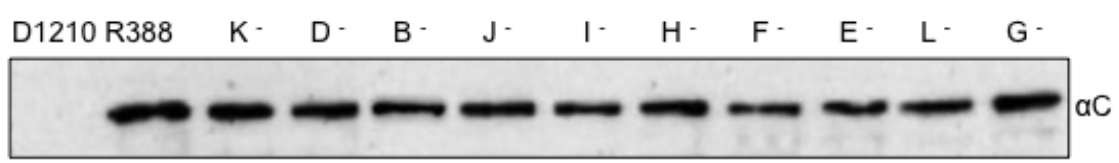

B

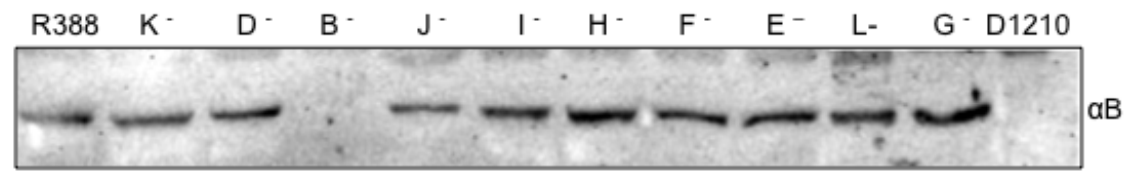

Figure S1. Western blots to detect TrwB and TrwC. Total extracts of E. coli cells containing R388 mutants were processed as described in Materials and Methods. Samples were run on SDS-PAGE, and proteins were detected with A) anti-TrwC $(\alpha C)$, or B) anti-TrwB ( $\alpha B)$. At the top of the gel, the Trw mutant is indicated. D1210: E. coli cells with no plasmid. 
Table 1. Plasmids used in this work

\begin{tabular}{|c|c|c|c|}
\hline Plasmid & Phenotype & Description & Reference \\
\hline $\mathrm{pAB2}$ & $K m^{R} T^{R}$ & pLAFR5-Km: : trw T4SS (Bt) & (Seubert et al., 2003) \\
\hline pDEL003 & $\mathrm{Cm}^{\mathrm{R}}$ & pSU19: : trwA-trwB & (de Paz et al., 2010) \\
\hline pHP138 & $\mathrm{Km}^{\mathrm{R}}$ & pET29c: : RLX (R388) & (de Paz et al., 2010) \\
\hline pHP139 & $\mathrm{Cm}^{\mathrm{R}}$ & pSU19: : trwB & (de Paz et al., 2010) \\
\hline pKM101 & $A p^{R}$ & Natural plasmid, IncN & $\begin{array}{l}\text { (Martínez and de la } \\
\text { Cruz, 1988) }\end{array}$ \\
\hline $\mathrm{pKM} 101 \Delta \mathrm{mob}$ & $A p^{R}$ & pKM101 D Dtr (T4SS only) & (Draper et al., 2005) \\
\hline pMTX539 & $A p^{R}$ & pHG329: RLX (pKM101) & (Llosa et al., 2003) \\
\hline pSU1443 & $K m^{R} T^{R}$ & R388: :Tn5tac1 in trwB & (Llosa et al., 1994) \\
\hline pSU2007 & $\mathrm{Km}^{\mathrm{R}}$ & $\mathrm{R} 388 \mathrm{Km}^{\mathrm{R}}$ & $\begin{array}{l}\text { (Martínez and de la } \\
\text { Cruz, 1988) }\end{array}$ \\
\hline pSU4028 & $\mathrm{Cm}^{\mathrm{R}}$ & $\begin{array}{l}\text { p15A: : R388 TRA with } \Omega \\
\text { insertion in trwA }\end{array}$ & (Bolland et al., 1990) \\
\hline pSU4035 & $\mathrm{Cm}^{\mathrm{R}}$ & $\begin{array}{l}\text { p15A: : R388 TRA with } \Omega \\
\text { insertion in trwG }\end{array}$ & (Bolland et al., 1990) \\
\hline pSU4058 & $A p^{R}$ & pHG327: : trwL-trwD & (Bolland et al., 1990) \\
\hline pSU4130 & $K m^{R} T p^{R}$ & R388: :Tn5tac1 in trwL & (de Paz et al., 2005) \\
\hline pSU4131 & $K m^{R} T p^{R}$ & R388: :Tn5tac1 in trwH & (Bolland, 1991) \\
\hline pSU4132 & $K m^{R} T p^{R}$ & R388: :Tn5tac1 in trwD & (Bolland, 1991) \\
\hline pSU4133 & $K m^{R} T^{R}$ & R388: :Tn5tac1 in trwK & (Llosa et al., 2003) \\
\hline pSU4134 & $K m^{R} T^{R}$ & R388: :Tn5tac1 in trwE & (Llosa et al., 2003) \\
\hline pSU4135 & $K m^{R} T p^{R}$ & R388: :Tn5tac1 in trwF & (de Paz et al., 2005) \\
\hline pSU4136 & $K m^{R} T p^{R}$ & R388: :Tn5tac1 in trwJ & (de Paz et al., 2005) \\
\hline pSU4137 & $\mathrm{Km}^{\mathrm{R}} \mathrm{Tp}^{\mathrm{R}}$ & R388: :Tn5tac1 in trwl & (de Paz et al., 2005) \\
\hline
\end{tabular}


Table 2. Summary of the results from Western blot assays

\begin{tabular}{|c|c|c|c|c|c|c|c|}
\hline $\begin{array}{c}\text { Trw R388 } \\
\text { T4SS }\end{array}$ & $\begin{array}{c}\text { Trw Bt } \\
\text { T4SS }\end{array}$ & DNA transfer & Anti C & Anti B & Anti D & Anti $\mathrm{K}$ & Anti J \\
\hline All & & +++ & ++ & ++ & ++ & ++ & ++ \\
\hline - TrwB (VirD4) & - & - & ++ & - & + & ++ & +++ \\
\hline - TrwD (VirB11) & - & - & ++ & ++ & - & +++ & - \\
\hline - TrwD (VirB11) & $\checkmark$ & +++ & & & - & & +++ \\
\hline - TrwK (VirB4) & - & - & ++ & ++ & - & - & - \\
\hline -TrwK (VirB4) & $\checkmark$ & - & & & ++ & & - \\
\hline - TrwE (VirB10) & - & - & ++ & ++ & ++ & +++ & - \\
\hline - TrwE (VirB10) & $\checkmark$ & + & & & +++ & & +++ \\
\hline - TrwG (VirB8) & - & & ++ & ++ & - & + & - \\
\hline - TrwG (VirB8) & $\checkmark$ & + & & & - & & + \\
\hline - TrwI (VirB6) & - & - & ++ & ++ & + & + & - \\
\hline - TrwI (VirB6) & $\checkmark$ & - & & & + & & - \\
\hline - TrwF (VirB9) & - & - & ++ & ++ & - & + & - \\
\hline - TrwF (VirB9) & $\checkmark$ & + & & & - & & +++ \\
\hline - TrwH (VirB7) & - & + & ++ & ++ & +++ & +++ & - \\
\hline - TrwH (VirB7) & $\checkmark$ & ++ & & & +++ & & +++ \\
\hline - TrwJ (VirB5) & - & - & ++ & ++ & +++ & +++ & - \\
\hline - Trw] (VirB5) & $s$ & - & & & +++ & & - \\
\hline
\end{tabular}

First column: the letter indicates the missing R388 Trw protein. Second column: the presence $(V)$ or absence (-) of Bt Trw-T4SS is indicated. Third column: DNA transfer frequencies (from de Paz et al., 2005):,$+++ 10^{-1}-10^{-2} ;++, 10^{-2}-10^{-4} ;+, 10^{-4}-10^{-6} ;-,<10^{-6}$ transconjugants/donor. The rest of columns show the steady-state of the proteins observed with each anti-Trw serum. ++: wild type levels; + decreased levels; - not detected; +++ increased levels compared to wild type. 


\section{References}

Alegria, M. C., et al., 2005. Identification of new protein-protein interactions involving the products of the chromosome- and plasmid-encoded type IV Secretion Loci of the Phytopathogen Xanthomonas axonopodis pv. citri. J Bacteriol. 187, 2315-25.

Alvarez-Martínez, C. E., Christie, P. J., 2009. Biological diversity of prokaryotic type IV secretion systems. Microbiol Mol Biol Rev. 73, 775-808.

Atmakuri, K., et al., 2004. Energetic components VirD4, VirB11 and VirB4 mediate early DNA transfer reactions required for bacterial type IV secretion. Mol Microbiol. 54, 1199-211.

Baron, C., et al., 1997. The lipoprotein VirB7 interacts with VirB9 in the membranes of Agrobacterium tumefaciens. J Bacteriol. 179, 1211-8.

Bolland, S., 1991. Genes implicados en la producción del pilus conjugativo de R388. Ph.D. Thesis. Universidad de Cantabria, Santander.

Bolland, S., et al., 1990. General organization of the conjugal transfer genes of the IncW plasmid R388 and interactions between R388 and IncN and IncP plasmids. J Bacteriol. 172, 5795-802.

Cabezon, E., de la Cruz, F., 2006. TrwB: an F(1)-ATPase-like molecular motor involved in DNA transport during bacterial conjugation. Res Microbiol. 157, 299-305.

Cascales, E., Christie, P. J., 2004a. Definition of a bacterial type IV secretion pathway for a DNA substrate. Science. 304, 1170-3.

Chandran, V., et al., 2009. Structure of the outer membrane complex of a type IV secretion system. Nature. 462, 1011-5.

Christie, P. J., et al., 2005. Biogenesis, architecture, and function of bacterial type iv secretion systems. Annu Rev Microbiol. 59, 451-85.

Christie, P. J., Cascales, E., 2005. Structural and dynamic properties of bacterial type IV secretion systems (review). Mol Membr Biol. 22, 51-61.

Das, A., et al., 1997. Delineation of the interaction domains of Agrobacterium tumefaciens VirB7 and VirB9 by use of the yeast two-hybrid assay. J Bacteriol. 179, 3404-9.

de la Cruz, F., et al., 2010. Conjugative DNA metabolism in Gram-negative bacteria. FEMS Microbiol Rev. 34, 18-40.

de Paz, H. D., et al., 2010. Functional dissection of the conjugative coupling protein TrwB. J Bacteriol. 192, 2655-2669.

de Paz, H. D., et al., 2005. Functional interactions between type IV secretion systems involved in DNA transfer and virulence. Microbiology. 151, 350516.

Draper, 0., et al., 2005. Site-specific recombinase and integrase activities of a conjugative relaxase in recipient cells. Proc Natl Acad Sci U S A. 102, 1638590.

Farizo, K. M., et al., 1996. Evidence for a ninth gene, $p t l I$, in the locus encoding the pertussis toxin secretion system of Bordetella pertussis and formation of a PtlI-PtlF complex. J Biol Chem. 271, 31643-9.

Fernandez-Lopez, R., et al., 2006. Dynamics of the IncW genetic backbone imply general trends in conjugative plasmid evolution. FEMS Microbiol Rev. 30, 942-66. 
Fernandez, D., et al., 1996b. The Agrobacterium tumefaciens virB7 gene product, a proposed component of the T-complex transport apparatus, is a membraneassociated lipoprotein exposed at the periplasmic surface. J Bacteriol. 178, 3156-67.

Fronzes, R., et al., 2009. The structural biology of type IV secretion systems. Nat Rev Microbiol. 7, 703-14.

Garcillan-Barcia, M. P., et al., 2009. The diversity of conjugative relaxases and its application in plasmid classification. FEMS Microbiol Rev. 33, 657-87.

Ghosh, A., et al., 2008. A hybrid vector system expands adeno-associated viral vector packaging capacity in a transgene-independent manner. Mol Ther. 16, 124-30.

Gilmour, M. W., et al., 2003. Interaction between the IncHI1 plasmid R27 coupling protein and type IV secretion system: TraG associates with the coiled-coil mating pair formation protein TrhB. Mol Microbiol. 49, 105-16.

Grandoso, G., et al., 1994. Purification and biochemical characterization of TrwC, the helicase involved in plasmid R388 conjugal DNA transfer. Eur J Biochem. 226, 403-12.

Guglielmini, J., et al., 2013. Evolution of Conjugation and Type IV Secretion Systems. Mol Biol Evol. 30, 315-331.

Hapfelmeier, S., et al., 2000. VirB6 is required for stabilization of VirB5 and VirB3 and formation of VirB7 homodimers in Agrobacterium tumefaciens. J Bacteriol. 182, 4505-11.

Hoppner, C., et al., 2004. VirB1 orthologs from Brucella suis and pKM101 complement defects of the lytic transglycosylase required for efficient type IV secretion from Agrobacterium tumefaciens. J Bacteriol. 186, 1415-22.

Krall, L., et al., 2002. Detergent extraction identifies different VirB protein subassemblies of the type IV secretion machinery in the membranes of Agrobacterium tumefaciens. Proc Natl Acad Sci U S A. 99, 11405-10.

Lai, E. M., et al., 2000a. Genetic and environmental factors affecting T-pilin export and T-pilus biogenesis in relation to flagellation of Agrobacterium tumefaciens. J Bacteriol. 182, 3705-16.

Lawley, T. D., et al., 2002. Functional and mutational analysis of conjugative transfer region 1 (Tra1) from the IncHI1 plasmid R27. J Bacteriol. 184, 2173-80.

Llosa, M., et al., 1994. Genetic organization of the conjugal DNA processing region of the IncW plasmid R388. J Mol Biol. 235, 448-64.

Llosa, M., de la Cruz, F., 2005. Bacterial conjugation: a potential tool for genomic engineering. Res Microbiol. 156, 1-6.

Llosa, M., et al., 2002. Bacterial conjugation: a two-step mechanism for DNA transport. Mol Microbiol. 45, 1-8.

Llosa, M., et al., 1991. Tn5tac1 insertion polarity in Escherichia coli. Plasmid. 26, 222-4.

Llosa, M., et al., 2003. Conjugative coupling proteins interact with cognate and heterologous VirB10-like proteins while exhibiting specificity for cognate relaxosomes. Proc Natl Acad Sci U S A. 100, 10465-70.

Martínez, E., de la Cruz, F., 1988. Transposon Tn21 encodes a RecA-independent site-specific integration system. Mol Gen Genet. 211, 320-5.

Peña, A., et al., 2011. Autoinhibitory regulation of TrwK, an essential VirB4 ATPase in type IV secretion systems. J Biol Chem. 286, 17376-82. 
Rivas, S., et al., 1997. TrwD, a protein encoded by the IncW plasmid R388, displays an ATP hydrolase activity essential for bacterial conjugation. J Biol Chem. 272, 25583-90.

Sagulenko, E., et al., 2001a. Role of Agrobacterium VirB11 ATPase in T-pilus assembly and substrate selection. J Bacteriol. 183, 5813-25.

Sambrook, J., Russell, D. W., 2001. Molecular cloning : a laboratory manual. Cold Spring Harbor Laboratory Press, Cold Spring Harbor, N.Y., p. 3 v.

Sandler, S. J., Clark, A. J., 1990. Factors affecting expression of the recF gene of Escherichia coli K-12. Gene. 86, 35-43.

Sastre, J. I., 1996. El extremo carboxilo de la proteína TraD del plásmido F confiere especificidad y eficiencia en el proceso de la conjugación. Ph. D. Thesis., Universidad de Cantabria.

Schmidt-Eisenlohr, H., et al., 1999b. TraC of IncN plasmid pKM101 associates with membranes and extracellular high-molecular-weight structures in Escherichia coli. J Bacteriol. 181, 5563-71.

Seubert, A., et al., 2003. A bacterial conjugation machinery recruited for pathogenesis. Mol Microbiol. 49, 1253-66.

Shamaei-Tousi, A., et al., 2004. Interaction between protein subunits of the type IV secretion system of Bartonella henselae. J Bacteriol. 186, 4796-801.

Tato, I., et al., 2007. The ATPase activity of the DNA transporter TrwB is modulated by protein TrwA: implications for a common assembly mechanism of DNA translocating motors. J Biol Chem. 282, 25569-76.

Towbin, H., et al., 1979. Electrophoretic transfer of proteins from polyacrylamide gels to nitrocellulose sheets: procedure and some applications. Proc Natl Acad Sci U S A. 76, 4350-4.

Waksman, G., Fronzes, R., 2010. Molecular architecture of bacterial type IV secretion systems. Trends Biochem Sci. 35, 691-8.

Wallden, K., et al., 2012. Structure of the VirB4 ATPase, alone and bound to the core complex of a type IV secretion system. Proc Natl Acad Sci U S A. 109, 11348-53.

Yuan, Q., et al., 2005. Identification of the VirB4-VirB8-VirB5-VirB2 pilus assembly sequence of type IV secretion systems. J Biol Chem. 280, 26349-59. 